\title{
Editorial
}

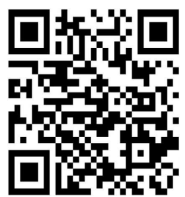

\section{Aspirin for primary prevention of cardiovascular events: what are the evidences?}

\author{
Yenny \\ Department of Pharmacology, Faculty of Medicine, Trisakti University
}

\begin{abstract}
Cardiovascular disease (CVD) is the leading cause of mortality in the United States, accounting for about 1 in 3 deaths. ${ }^{(1)}$ In view of the healthcare and economic burdens of cardiovascular disease, the public health aim should be the prevention of cardiovascular events through risk factor optimization.
\end{abstract}

Aspirin is essential for antiplatelet pharmacotherapy in patients with cardiovascular diseases because it plays a well-established role in secondary prevention and is widely available and affordable. It has been suggested that aspirin doses $<75 \mathrm{mg}$ daily may be more effective than higher doses because they spare prostacyclin (an antiplatelet and vasodilator) and cause less gastrointestinal toxicity. ${ }^{(2)}$ However, the use of aspirin in primary prevention of cardiovascular events is controversial. Several large scale placebo-controlled randomized studies have been conducted to determine the effect of low-dose aspirin $(100 \mathrm{mg} /$ day $)$ in the prevention of primary cardiovascular events, among others The Aspirin to Reduce Risk of Initial Vascular Event (ARRIVE) trial, ${ }^{(3)}$ A Study of Cardiovascular Events in Diabetes (ASCEND) ${ }^{(4)}$ and The Aspirin in Reducing Events in the Elderly (ASPREE) trial, ${ }^{(5)}$ with durations of $2.5,7.4$, and 4.7 years, respectively. Of the three above-mentioned studies, it was only ASCEND ${ }^{(4)}$ that showed the efficacy of aspirin in the prevention of primary cardiovascular events, i.e. in subjects with moderate cardiovascular risks. The increase in risk of bleeding in the group of subjects using aspirin was reported in all three studies.
To date there is no strong evidence with regard to the specific dose of aspirin to be used in primary prevention of cardiovascular events, but the low dose aspirin used in in clinical practice is 75 to $100 \mathrm{mg} /$ day. The use of higher doses does not increase the efficacy, but increases the risk of gastrointestinal hemorrhage.

A meta-analysis carried out by Lin et al. ${ }^{(6)}$ showed that the dose of aspirin used in studies on the effect of aspirin in primary cardiovascular prevention ranges from $100 \mathrm{mg}$ every other day to $650 \mathrm{mg}$ per day. This is due to the fact that even though the anti-thrombotic effect of aspirin is apparently not dose- related, since inhibition of platelet COX-1 by aspirin is saturated at low doses, the gastrointestinal toxicity is doserelated. ${ }^{(7)}$

The controversy on the results of the largescale studies is also reflected in the various guidelines used in clinical practice. The European Society of Cardiology (ESC) guideline ${ }^{(8)}$ does not recommend the use of aspirin for primary prevention of cardiovascular events. The US Preventive Service Task Force guideline ${ }^{(9)}$ recommends the use of aspirin in individuals 5059 years of age who have a 10 -year risk of CV disease of $\geq 10 \%$. The American College of Cardiology/American Heart Association (ACC/ AHA) Task Force on Clinical Practice guideline ${ }^{(10)}$ recommends aspirin only in individuals with atherosclerotic cardiovascular disease (ASCVD) ages 40-70 years who are at high risk for ASCVD, but are not at high risk for gastrointestinal hemorrhage. The American College of 
Cardiology/American Heart Association do not recommend aspirin in subjects $>70$ years of age, or at any age who are at high risk for hemorrhage.

Based on existing evidence the benefit of low-dose aspirin in the prevention of primary cardiovascular events is still a controversial subject that impacts on the variety of existing clinical guidelines. In a meta-analysis of 13 trials with 164,225 participants without cardiovascular disease, aspirin use was associated with a lower risk of cardiovascular events, defined as cardiovascular death, nonfatal myocardial infarction, and nonfatal stroke (hazard ratio [HR], 0.89; absolute risk reduction, $0.41 \%$ ) and an increased risk of major bleeding (HR, 1.43; absolute risk increase, $0.47 \%){ }^{(11)}$

From a practical point of view, should healthy people take a daily aspirin? The logical answer should be no, unless recommended by your physician. Although it is an inexpensive over-thecounter drug, aspirin may cause bleeding, the risk increasing with age. Therefore, healthy individuals with no history of heart disease should not just start taking aspirin without medical advice. However, for healthy individuals at very high risk of heart attack, based on current smoking, family history of premature heart attacks, or very high cholesterol level with intolerance to statins, the preventive use of low-dose aspirin might be justified, after consultation with the cardiologist.

\section{REFERENCES}

1. Mozaffarian D, Benjamin EJ, GoAS, et al. American Heart Association Statistics Committee and Stroke Statistics Subcommittee Heart disease and stroke statistics - 2015 update: a report from the American Heart Association. Circulation 2015; 131:e29-322. doi: 10.1161/CIR.0000000000000 152.

2. Gargiulo G, Windecker S, Vranckx P, Gibson CM, Mehran R, Valgimigli M. A critical appraisal of aspirin in secondary prevention: is less more? Circulation 2016;134:1881-1906. doi: 10.1161/ CIRCULATIONAHA.116.023952.

3. Gaziano JM, Brotons C, Coppolecchia R, et al. Use of aspirin to reduce risk of initial vascular events in patients at moderate risk of cardiovascular disease (ARRIVE): a randomised, double-blind, placebo-controlled trial. Lancet 2018;392:1036-46. doi: 10.1016/S0140-6736(18) 31924-X.

4. Bowman L, Mafham M, Wallendszus K, et al. Effects of aspirin for primary prevention in persons with diabetes mellitus. New Engl J Med 2018;379:1529-39. doi: 10.1016/j.jvs.2018.10.072.

5. McNeil JJ, Wolfe R, Woods RL, et al. Effect of aspirin on cardiovascular events and bleeding in the healthy elderly. New Engl J Med 2018;379:1509-18. doi: 10.1056/NEJMoa1805819.

6. Lin MH, Lee CH, Lin C, et al. Low-dose aspirin for the primary prevention of cardiovascular disease in diabetic individuals: a meta-analysis of randomized control trials and trial sequential analysis. J Clin Med 2019;8:pii.E609. doi: 10.3390/ jcm8050609.

7. Rothwell PM, Cook NR, Gaziano JM, et al. Effects of aspirin on risks of vascular events and cancer according to bodyweight and dose: analysis of individual patient data from randomised trials. Lancet 2018;392:387-99. DOI: https://doi.org/ 10.1016/S0140-6736(18)31133-4.

8. Piepoli MF, Hoes AW, Agewall S, et al. European guidelines on cardiovascular disease prevention in clinical practice: The Sixth Joint Task Force of the European Society of Cardiology and Other Societies on Cardiovascular Disease Prevention in Clinical Practice (constituted by representatives of 10 societies and by invited experts) Developed with the special contribution of the European Association for Cardiovascular Prevention \& Rehabilitation (EACPR). Eur Heart J 2016;37:2315-81. doi: 10.1093/eurheartj/ehw106.

9. Bibbins-Domingo K. US Preventive Services Task Force. Aspirin use for the primary prevention of cardiovascular disease and colorectal cancer: US Preventive Services Task Force Recommendation Statement. Ann Inter Med 2016;164:836-45. doi: 10.7326/M16-0577.

10. Arnett DK, Blumenthal RS, Albert MA, et al. 2019 ACC/AHA Guideline on the primary prevention of cardiovascular disease. Circulation 2019: CIR0000000000000677. doi: 10.1161/CIR. 0000000000000677.

11. Zheng SL, Alistair J. Roddick AJ. Association of aspirin use for primary prevention with cardiovascular events and bleeding events: a systematic review and meta-analysis. JAMA 2019; 321:277-87. doi: 10.1001/jama.2018.20578. 